\title{
Bright Picosecond X-rays from Intense Sub-Picosecond Laser-Plasma Interactions
}

\author{
A. Maksimchuk, J. Workman, X. Liu, U. Ellenberger, \\ S. Coe, C.-Y. Chien, and D. Umstadter \\ Center for Ultrafast Optical Science, \\ University of Michigan, Ann Arbor, MI 18109-2099 \\ Phone: (313) 764-2284, Fax: (\$13) 763-4876
}

\begin{abstract}
Short-pulse, high-intensity laser-plasma interactions are investigated experimentally with temporally and spectrally resolved soft $x$-ray diagnostics. The emitted x-ray spectra from solid targets of various $Z$ are characterized for a range of laser intensities $\left(I<5 \times 10^{17} \mathrm{~W} / \mathrm{cm}^{2}\right)$ and pulse widths $\left(\pi_{l} \sim 400\right.$ fs). With low contrast $\left(10^{5}\right)$, the $x$-ray spectrum in the $\lambda=40-100 \AA$ spectral region is dominated by line emission, and the $x$-ray pulse duration is found to be long, $\tau_{x}>100 \mathrm{ps}$, characteristic of a long-scale-length, low-density plasma. Bright, picosecond, continuum emission, characteristic of a short-scalelength, high-density plasma, is produced only when a high laser contrast $\left(10^{10}\right)$ is used. It is demonstrated experimentally that the pulsewidth of laser-produced $x$-ray radiation may be varied down to the picosecond time-scale by adjusting the incident ultrashort-pulse laser flux. This controls the peak electron temperature relative to the ionization potential, corresponding to the emitted $\mathrm{x}$-ray photon energy of interest. The results are found to be consistent with the predictions of a hydrodynamics code coupled to an average atom model only if non-local thermodynamic equilibrium (NLTE) is assumed.
\end{abstract}

Short-pulse high-intensity lasers interacting with solid targets make possible the study of a new class of plasmas. They are unique because during the ultrashort laser pulse relatively little expansion occurs and the density scale length remains much less than the laser wavelength. This makes possible the deposition of a significant amount of the laser energy at densities much greater than the critical density. The $x$-ray pulse will be extremely bright because of the high density, the small dimensions of the laser spot size, and the ultrashort $x$-ray pulse width. The latter is due to rapid cooling by expansion and diffusion, which results from the steep temperature and density gradients, and the high collision rates at high density. These bright compact sources of ultrashort-pulses of $\mathrm{x}$-rays have applications in time-resolved diffraction, holography, spectroscopy, microscopy or radiography studies of transient biological or physical phenomena.

Picosecond $\mathrm{x}$-ray emission from laser-produced plasmas has been demonstrated in previous investigations. Murnane et al. [1] observed, using a filtered x-ray streak camera, that the laser contrast strongly affects the $x$-ray pulse width: the higher the 
contrast, the shorter the pulse width. These results were attributed to the fact that only with high contrast can the laser energy be deposited at high plasma density. Using high-resolution crystal spectroscopy in the keV region, Kieffer et al. [2] and Riley et al. [3] inferred the existence of a solid density plasma from x-ray line widths.

In this paper, we demonstrate the important effects of the laser intensity $(I)$ on the $x$-ray pulsewidth. Time-resolved x-ray spectra from an intense subpicosecond laser interacting with solid targets of aluminum and gold are characterized with a high temporal and spectral resolution. Bright picosecond continuum emission in the 20-300 $\AA$ spectral region, characteristic of a solid density plasma, is measured only when a high laser contrast is used. Otherwise, longer pulse line-radiation, characteristic of a critical density plasma, dominates the emission. It is demonstrated experimentally for the first time that the $\mathrm{x}$-ray pulse duration can be controlled by appropriately adjusting $I$, and thus the average electron temperature $T_{e}$. The plasma will generate $x$-ray line emission characteristic of the average ionization stage $\bar{Z}$ corresponding to $T_{0}$ when $T_{e} \sim T_{0}$, where $T_{0}$ is an "ionization temperature," which in equilibrium is taken to be approximately one third of the ionization potential for that stage. A given region of the plasma is heated to $T_{0}$ either by direct deposition of laser energy or diffusion of heat from neighboring regions. The rise-time of the $\mathrm{x}$-ray pulse is determined by the time it takes to heat the region where the greatest emission occurs. The fall-time is given by the cooling rate, which should decrease exponentially because it is driven by the density and temperature gradients, both of which decrease as the plasma cools either by expansion into the vacuum, or by heat conduction into the colder regions of the solid.

The x-ray pulse should be extremely short when $I$ is low, such that the peak $T_{e}\left(T_{\max }\right)$ just barely reaches $T_{0}$ and then rapidly decreases below $T_{0}$. At higher $I$ such that $T_{\max }>T_{0}$, the plasma emission coressponding to $T_{0}$ will continue until $T_{e}$ drops below $T_{0}$ [4]. However, at later times, the gradients relax, and, consequently, so does the slope of the exponential decrease in emission.

The laser used in the experiment is a 400 -fs terawatt $\mathrm{Nd}$-glass laser system based on chirped pulse amplification with a contrast ratio of the fundamental $1.06 \mu \mathrm{m}$ laser light measured to be $5 \times 10^{5}$ and increased to $10^{10}$ by frequency doubling. An offaxis parabolic mirror is used to focus the laser radiation on a solid target at normal incidence to a minimum spot size of $15 \mu \mathrm{m}$, corresponding to a maximum intensity in $2 \omega$ of $5 \times 10^{17} \mathrm{~W} / \mathrm{cm}^{2}$. In order to decrease the incident flux on the target the laser spot size was defocused while keeping the total laser energy constant. The soft $\mathrm{x}$-ray emission is dispersed using an imaging flat-field grazing-incidence variablespaced grating spectrometer. Time-resolved spectra are obtained in a single shot using a streak camera, coupled to the spectrometer, with a 5-ps resolution that is read-out with a CCD camera. An x-ray pinhole camera, filtered with $25 \mu \mathrm{m}$ of $\mathrm{Be}$ and coupled to an intensified microchannel plate detector, was used to monitor the laser spot size. Two absolutely calibrated pin diodes filtered with $50 \mu \mathrm{m}$ and 100 $\mu \mathrm{m}$ of $\mathrm{Be}$ were used to monitor $\mathrm{keV} \mathrm{x}$-ray emission levels. To receive information about conversion efficiency of the laser radiation into $x$-rays in the range $1.5-5 \mathrm{keV}$ we used DEF-film with steps of Be-filters of different thicknesses and with known characteristic curves [5]. 
Figure 1 shows the emission using $1.06 \mu \mathrm{m}$ laser irradiation on an aluminum target $(Z=13)$, corresponding to low-contrast conditions. The central plot is a single-shot streak picture showing the $\mathrm{x}$-ray amplitude (grayscale in units of CCD counts) versus wavelength (vertical axis in units of $\AA$ ) and time (horizontal axis in units of picoseconds). To the left is a line-out in wavelength at the time of peak emission, and at the bottom is a line-out in time for $\lambda=55 \AA$ ( $h \nu=225$ $\mathrm{eV}$ ), corresponding to the $2 s 2 p-2 s 3 d$ transition in $\mathrm{ALX}$. It can be seen that the emission is dominated by lines and that the pulse duration is long, $\tau \sim 70$ ps (FWHM). Figure 1(b) shows aluminum emission using $2 \omega(0.53 \mu \mathrm{m})$ irradiation, corresponding to high-contrast conditions. To the left is a line-out in wavelength at the peak of emission, and at the bottom is a line-out in time for $\lambda=55 \AA$. Note that in the high-contrast case the emission is continuum dominated over the entire wavelength range and that the pulse duration $(\tau \sim 25 \mathrm{ps})$ is shorter than in the low-contrast case. In both figures, the sharp cutoff near the C K-edge ( $44 \AA$ ) is due to absorption by the lexan film that supports the potassium bromide photocathode. The falltime is faster for the high-contrast $2 \omega$ illumination case, presumably because the plasma density scale length is shorter and also the critical density is higher.
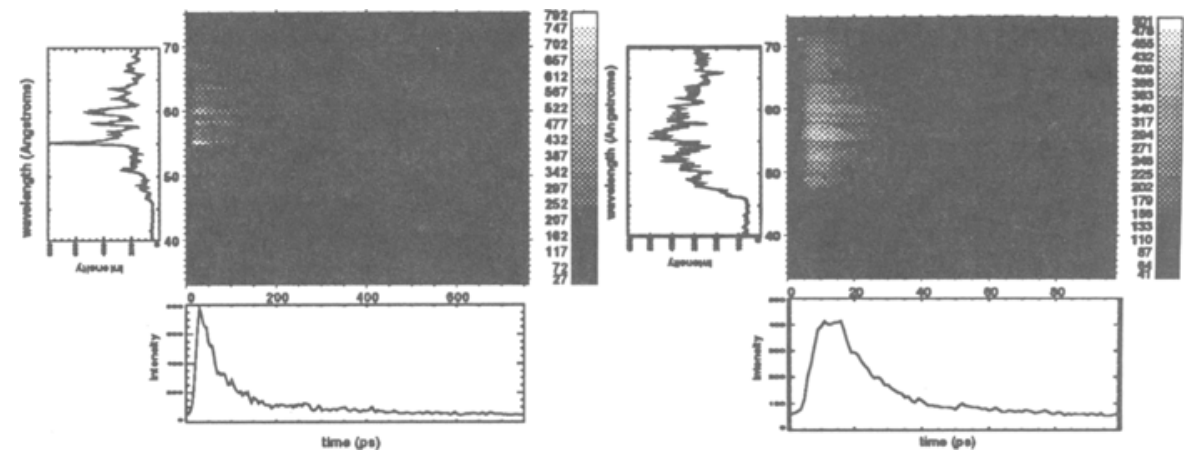

Figure 1: Streaked aluminum $\mathrm{x}$-ray spectra obtained in a single shot with a highresolution grazing-incidence spectrometer using (a) $\omega$ laser irradiation, corresponding to low-contrast conditions, and (b) using $2 \omega$ laser irradiation, corresponding to high-contrast conditions.

Figure 2(a) shows a comparison of amplitude-normalized temporal profiles of the AlXI $1 s^{2} 2 s-1 s^{2} 3 p$ line $(\lambda=48.3 \AA)$ obtained for $2 \omega$ irradiation for a range of laser flux densities. Note the pronounced decrease of the x-ray pulse width as $I$ is decreased. In the low- $I$ case, the FWHM of the X-ray pulse appears to be streak camera limited. The risetime is short in all cases (the starting positions of the plots relative to the laser pulse and each other are unknown.) The falltime is observed to be shortest in duration in the lowest $I$ case as expected from the previous arguments.

Figure 2(b) shows a similar behavior of amplitude-normalized temporal profiles of the same line $(\lambda=48.3 \AA)$ as predicted by a hydrodynamics code coupled to an atomic physics code for a range of laser flux densities. The one-dimensional hydro- 

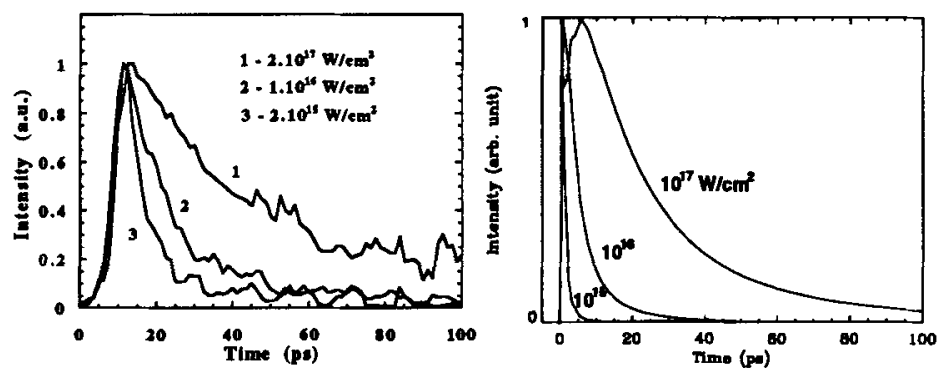

Figure 2: Comparison of amplitude-normalized temporal profiles obtained experimentally (a) and predicted by the numerical code (b).

dynamics code solves the conservation equations and is coupled self-consistently to the Helmholtz equation of the laser field for energy and momentum deposition in the plasma. A non-local thermodynamic equilibrium (NLTE) detailed-configuration time-dependent atomic physics package, FLY, is used to calculate the ionization and spectral emission [6,7]. Figure 3(a) shows the comparison of measured and simulated $x$-ray pulse duration as a function of laser flux. The averaged experimental aluminum pulse widths at $\lambda=48.3 \AA$ are shown as crosses. The predicted spatially integrated values for aluminum at this wavelength are shown as pluses and fit with a spline routine. The dashed curve represents the predicted results convolved with the 5-ps response of the $\mathrm{x}$-ray streak camera. Discrepancies between the experimental data and theory may possibly be due in part to opacity effects not included in the model at this time. The model does, however, give us some insight into the physics controlling the pulse duration. The hydro simulation reveals that the electron temperature rises very quickly, as expected, but falls on a time scale that is much faster than that of the $x$-ray pulse duration. We see that the $x$-ray pulse duration does not follow the temperature time history profile. The reason for this becomes clear when we look at the time history of $\bar{Z}$, which remains high long after $T_{e}$ has dropped. The plasma is thus NLTE, and therefore the $\mathrm{x}$-ray emission is no longer dominated by the temperature but by a combination of the electron temperature, electron density and average ionization. X-ray pulse durations at $\lambda=48.3 \AA$ were also measured for a gold target in order to compare the possible differences in brightness from different $\mathrm{Z}$ materials. For the same laser intensity the gold $\mathrm{x}$-ray pulse duration was found to be as little as half the aluminum pulse duration. Data on conversion efficiency of laser radiation into $x$-rays in the range $1.5-5 \mathrm{keV}$ are shown on figure $3(\mathrm{~b})$. Note that conversion efficiency for the gold target is about 4 times higher than for the aluminum target. For the highest laser fluxes on target the conversion efficiency can reach about 1 percent (a few $\mathrm{mJ}$ ) for the x-ray photons with $h \nu \geq 1 \mathrm{keV}$.

To summarize, we have shown that the pulse width of a given range of photon energies of continuum soft $x$-rays may be minimized by adjusting the laser focal spotsize, and thereby the incident laser intensity, such that $T_{\max } \leq T_{0}$. The results 

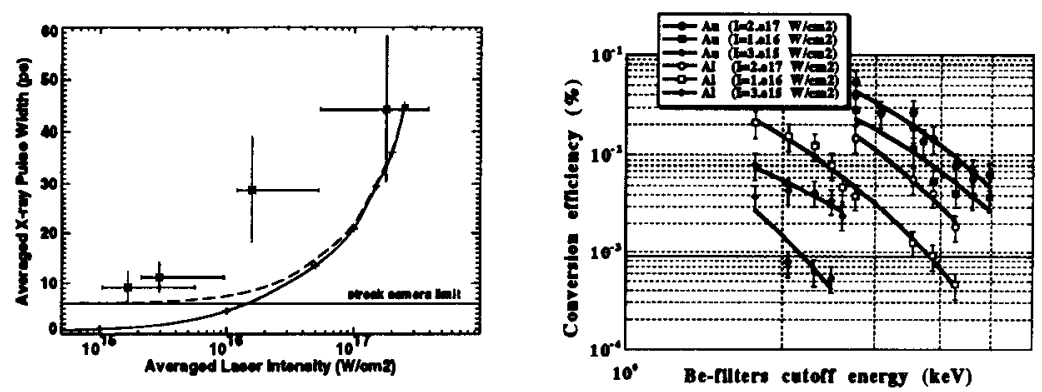

Figure 3: (a) Duration of the $\operatorname{AlX} I s^{2} 2 s-1 s^{2} 3 p$ line $(\lambda=48.3 \AA)$ versus laser intensity: experimental (crosses), predicted by the numerical code (solid line w/ pluses), predicted by the numerical code with convolution of streak camera response (dotted line). (b) Measured $\mathrm{x}$-ray conversion efficiency for aluminum and gold solid targets for a range of laser fluxes.

are found to be in close agreement with the predictions of the hydrodynamics code coupled to the atomic physics model only when NLTE effects are included. The conversion efficiency of the high contrast subpicosecond laser pulses into ultrashort soft $\mathrm{x}$-ray pulses can be as high as a few percent. We observed that the emission from gold targets in the $x$-ray region of $50 \AA$ is an order of magnitude brighter than the emission from the aluminum due both to its shorter pulse duration and higher $\mathrm{x}$-ray yield.

This work was partially funded by the National Science Foundation Center for Ultrafast Optical Science, contract \#PHY8920108, and Office of Naval Research, contract \#N00014-91-K-2005. The authors would like to thank G.Mourou for useful discussions and his encouragement.

\section{References}

[1] M. M. Murnane et al., Phys. Rev. Lett. 62, 155 (1989).

[2] J. C. Kieffer et al., Bull. Am. Phys. Soc. 37, 1468 (1992).

[3] D. Riley et al., Phys. Rev. Lett. 69, 3739 (1992).

[4] D. Umstadter et al., Bull. Amer. Phys. Soc. 34, 1364 (1989);

H. Milchberg et al., Phys. Rev. Lett. 67, 2654 (1991).

[5] B.L.Henke et al., J. Opt. Soc. Am. B 3, No.11, 1540 (1986).

[6] S.J.Rose, J.Quant. Spectrosc. Radiat. Transfer 36, 389 (1986).

[7] R.W.Lee et al., J.Quant. Spectrosc. Radiat. Transfer 32, 91 (1984). 